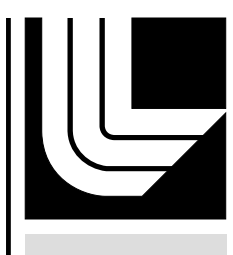

LAW RENCE LIVERMORE N A T IO N A L LABORATORY

On the Virial Theorem for

Interstellar Medium

D.D. Ryutov

September 25, 2007

Astrophysical Journal 
This document was prepared as an account of work sponsored by an agency of the United States Government. Neither the United States Government nor the University of California nor any of their employees, makes any warranty, express or implied, or assumes any legal liability or responsibility for the accuracy, completeness, or usefulness of any information, apparatus, product, or process disclosed, or represents that its use would not infringe privately owned rights. Reference herein to any specific commercial product, process, or service by trade name, trademark, manufacturer, or otherwise, does not necessarily constitute or imply its endorsement, recommendation, or favoring by the United States Government or the University of California. The views and opinions of authors expressed herein do not necessarily state or reflect those of the United States Government or the University of California, and shall not be used for advertising or product endorsement purposes. 


\title{
ON THE VIRIAL THEOREM FOR INTERSTELLAR MEDIUM
}

\author{
D. D. Ryutov \\ Lawrence Livermore National Laboratory, Livermore, CA 94551, USA
}

\begin{abstract}
An attempt has been made to derive a version of the virial integral that would describe average properties of the interstellar medium (ISM). It is suggested to eliminate the (large) contribution of stellar matter by introducing "exclusion zones" surrounding stars. Such an approach leads to the appearance of several types of additional surface integrals in the general expression. Their contribution depends on the rate of energy and matter exchange between the stars and ISM. If this exchange is weak, one can obtain a desired virial integral for ISM. However, the presence of intermittent large-scale energetic events significantly constrains the applicability of the virial theorem. If valid, the derived virial integral is dominated by cold molecular/atomic clouds, with only minor contribution of the global magnetic field and low-density warm part.
\end{abstract}

\section{INTRODUCTION}

The Claussius virial theorem (VT) is a powerful tool for the analysis of a variety of astrophysical systems. It helps in getting insights into their behavior without direct solution of the dynamical equations. The use of the virial theorem in stellar astrophysics is described in a comprehensive review by Collins (2003). An excellent presentation of the use of the virial theorem in the context of turbulent and magnetic effects in the evolution of molecular clouds was provided by McKee and Zweibel (1992). Both references contain also extensive bibliography.

In this article, the author considers the possibility of using the virial theorem for description of the interstellar medium (ISM) at a much greater spatial scale, roughly that of the thickness of a spiral arm (i.e., $\sim 1 \mathrm{kpc}$ ). We concentrate on the ISM in our Galaxy, at roughly a Solar circle.

The first issue that has to be addressed is an identification of a relatively welldefined subsystem which, on the one hand, could be considered as a dynamically isolated object and, on the other hand, would be large enough to make the time-averaging procedure meaningful. We argue that a good candidate for this purpose is a segment of the spiral arm with a length of order of its thickness. One might try to formulate the virial theorem for the whole Galaxy, which indeed can be treated as an isolated system, but this object is too nonuniform, even in the sense of averaged quantities, to make a virial theorem for the interstellar medium in it useful (and even feasible).

So, we consider a structure schematically shown in Fig. 1: an elongated segment of an arm, of a radius $R$, a few $R$ long, comprising stars and interstellar medium. The global rotation around the center of the Galaxy is eliminated by switching to the rotating frame. We neglect the rotation shear, because its characteristic time is somewhat longer than the characteristic dynamical time for establishing the "hydrostatic" radial equilibrium. 
In order to obtain the virial theorem in its standard form and eliminate difficult-toevaluate surface integrals that appear in the course of its derivation, we have to assume that the structure shown in Fig. 1 is well separated from the external medium not only in the radial but also in the axial direction. This is a rather severe limitation. [An alternative could be to consider not a segment of an arm, but an axisymmetric torus of a minor radius $R$; this system is, however, even more idealized than the one based on the assumption of a finite-length segment of a cylinder, separated by long enough gaps from the ambient medium.]

After having identified an isolated object shown in Fig. 1 that includes ISM and stars, we proceed to our attempt to derive the virial theorem for the interstellar medium. The difficulty in formulating the virial theorem for the ISM is related to the fact that the ISM is also not an isolated system but is strongly coupled with the stellar matter. If one considers the expression for the virial integral for the whole system, including both ISM and compact stellar objects, one finds that the main contribution to the virial integral comes from the gravitational and internal energy of the stellar matter, and this contribution exceeds by many orders of magnitude the contribution from ISM. Therefore the virial integral for the whole isolated system of Fig. 1 does not allow one to obtain any useful information regarding the properties of the ISM.

To overcome this difficulty, one can separate the contribution of the small volumes surrounding the stars from the total integration volume. We do that by introducing "exclusion zones" around stellar objects; the gravitational effect of the stellar matter on the dynamics of ISM remains then only in the form of smoothed gravitational potential.

After introducing exclusion zones, we are left with surface integrals over the interfaces between the exclusion zones and ISM. If these surface integrals are small and can be neglected, one obtains the desired virial integral for ISM, Sec. 3 .

However, the ISM itself is highly structured, with dense molecular and atomic clouds comprising a dominant part of the ISM mass. It turns out that they make a dominant contribution to the virial integral for the ISM, leaving very little for a more tenuous warm gas and global magnetic field. Therefore, the virial integral for ISM basically reduces to the sum over isolated individual dense clouds, occupying a fraction of the overall volume.

Despite this relatively negative conclusion, the author believes that the analysis presented in this paper is useful in revealing some aspects of the "inner workings" of the virial theorem.

It goes without saying that virial integrals for isolated clouds are still valid and quite useful for the analysis of the properties of individual clouds (Cf McKee \& Zweibel, 1992).

In the following, when making numerical estimates, we use the parameters characteristic of our Galaxy in the area near the solar circle (see reviews by Ferriere, 2001 and Lequeux, 2005): the average density of ISM $\rho_{\text {ISM }} \sim 3 \cdot 10^{-24} \mathrm{~g} / \mathrm{cm}^{3}, R \sim 300 \mathrm{pc}$, the average density of the stellar matter $\rho_{\text {st }}$ (when uniformly spread over the whole volume in question) $\sim 10 \rho_{\text {ISM }}$, the average energy density of ISM, with magnetic field energy and cosmic ray energy included, under the equipartition assumption, $W_{\text {ISM }} \sim 1 \mathrm{eV} / \mathrm{cm}^{3}$ (Ferriere, 2001; Lequeux, 2005). The dynamical time which we associate with the free- 
fall time over the thickness of the galactic disk can be evaluated as (Cf. Spitzer, 1978; Lequeux, 2005):

$t_{d y n} \sim 2 \cdot 10^{7}$ years

The inverse shear frequency is actually not much greater than $t_{d y n}$, but we don not attempt to address this additional difficulty here.

\section{A STANDARD VIRIAL THEOREM}

We use dynamic equations in the form:

$\rho \frac{d \mathbf{v}}{d t}=-\nabla p-\frac{1}{4 \pi}[\boldsymbol{B} \times \nabla \times \boldsymbol{B}]+\rho \boldsymbol{g}$,

$\frac{d \rho}{d t}+\rho \nabla \cdot \mathbf{v}=0$,

where $p$ and $\rho$ are the local pressure and the density, in particular, in the interior of the stars, $\mathbf{v}$ is the velocity, $\boldsymbol{B}$ is the magnetic field, and $\boldsymbol{g}$ is the gravity acceleration. The acceleration $\boldsymbol{g}$ is related to the density by the equations:

$g=-\nabla \Phi, \nabla^{2} \Phi=-4 \pi G \rho$,

where $G$ is the Newton's gravitational constant. Throughout the paper we use CGS system of units. As seen from Eqs. (2) and (3), we use the Eulerian description within which the parameters of the medium (say, its density $\rho$ ) are considered as functions of time and coordinates, $\rho=\rho(t, \boldsymbol{r})$, Cf. McKee and Zweibel, 1992.

One can use much more detailed level of description, based on kinetic equations, but this does not bring up any significant changes to our main conclusions as expressed by Eqs. (17) and (23) below.

By accounting for the continuity equation (3), one can transform Eq. (2) to the following divergent form:

$\frac{\partial \rho \mathrm{v}_{\alpha}}{\partial t}+\frac{\partial}{\partial x_{\beta}}\left(\rho \mathrm{v}_{\alpha} \mathrm{v}_{\beta}+p \delta_{\alpha \beta}\right)+\frac{\partial}{\partial x_{\beta}} \frac{\left(B^{2} \delta_{\alpha \beta}-B_{\alpha} B_{\beta}\right)}{8 \pi}=-\rho g_{\alpha}$

The standard (Einstein) summation rule over Greek indices is used. Multiplying this equation by $x_{\alpha}$, using the identity $x_{\alpha}\left(\partial A_{\alpha \beta} / \partial x_{\beta}\right) \equiv\left(\partial A_{\alpha \beta} x_{\alpha} / \partial x_{\beta}\right)-A_{\alpha \alpha}$ (where $A_{\alpha \beta}$ is an arbitrary tensor), and performing integration over the volume containing our system, one obtains the virial theorem in its standard form:

$\frac{d}{d t} \int_{V} \rho \mathrm{v}_{\alpha} x_{\alpha} d V+\int_{V}\left(\rho \mathrm{v}^{2}+3 p+\frac{B^{2}}{4 \pi}\right) d V-\int_{V} \rho g_{\alpha} x_{\alpha} d V=$

$-\int_{S}\left[\rho x_{\alpha} \mathrm{v}_{\alpha} \mathrm{v}_{\beta} d S_{\beta}-\frac{x_{\alpha} B_{\alpha} B_{\beta} d S_{\beta}}{4 \pi}+\left(p+\frac{B^{2}}{8 \pi}\right) x_{\beta} d S_{\beta}\right]$

where $S$ is the surface surrounding the integration volume. It goes without saying that this result, aside from notation and some re-arrangement of terms, coincides with the classical result of McKee and Zweibel, 1992 (see their Eq. (3.9)).

In our present analysis, we assume that the integration volume is well separated from the external medium. This allows us to place the surface in the zone with negligible density, pressure and magnetic field, thereby obtaining the virial theorem in its most traditional "volumetric" form: 
$\frac{d}{d t} \int_{V} \rho \mathrm{v}_{\alpha} x_{\alpha} d V+\int_{V}\left(\rho \mathrm{v}^{2}+3 p+\frac{B^{2}}{4 \pi}\right) d V-\int_{V} \rho g_{\alpha} x_{\alpha} d V=0$

If so desired, the last integral in the left-hand side (L.H.S.) can be presented in terms of the energy density of the gravitational field:

$\frac{d}{d t} \int_{V} \rho \mathrm{v}_{\alpha} x_{\alpha} d V+\int_{V}\left(\rho \mathrm{v}^{2}+3 p+\frac{B^{2}}{4 \pi}\right) d V-\frac{1}{8 \pi G} \int_{V} g^{2} d V=0$

where integration volume should be larger than the volume occupied by the matter, as $g$ is non-zero even in the area where $\rho=0$. As, at large distances from the system, the gravity acceleration scales inversely proportionally to the square of the distance, the last integral in Eq. (8) converges. The significance of the dynamic time-scale (Eq. (1)) is that this is a minimum time over which one has to average Eq. (7) or (8) to obtain a steadystate (time-averaged) version of the virial integral, provided the system is in the average equilibrium.

As has already been mentioned at the beginning of this paper, Eqs. (7) and (8) would not allow us to say much regarding the dynamics of the interstellar medium proper: both the pressure term and the gravitational energy term are dominated by the stellar matter. For reference purpose, we present an expression for the gravitational energy of a uniform sphere of a radius $a$ and mass $M_{s t}$. It is (Cf. Saslaw, 1985):

$W_{G}=\frac{3 G M_{s t}^{2}}{5 a}$

The gravitational energy of a standard star of 1 Solar mass $1 \mathrm{M}_{\odot}$ is, according to Eq. (9) $10^{60} \mathrm{eV}$. Assuming the average number of the stars per unit volume to be $\sim 0.1 / \mathrm{pc}^{3}$ (Ledoux, 2005), one finds that the energy of ISM in the volume containing one star is $\sim 10 \mathrm{pc}^{3} \times \mathrm{W}_{\mathrm{ISM}} \sim 3 \times 10^{55} \mathrm{eV}$, i.e., $4-5$ orders of magnitude less than the gravitational energy of the stellar matter in the same volume. We didn't try to be accurate in these estimates and presented them just to demonstrate that there is very large disparity between contributions of the stellar matter and ISM to Eq. (7). In particular, if one is interested in a quasi-steady state of ISM, one would have to guarantee an unrealistically precise $\left(10^{-5}\right)$ balance between the pressure terms and the gravity terms of the stellar matter.

One could argue that, when considering an individual star, one would have an almost exact cancellation of the positive (kinetic, internal, and magnetic energy) and negative (gravitational energy) contributions to the time-averaged integral. But this statement implies that the star is a perfectly isolated system, and the degree to which this assumption is valid is exactly the point that we are going to address.

\section{ELIMINATING THE STELLAR CONTRIBUTION}

To deal with this problem, one can try to eliminate the contribution of the zones surrounding the compact objects when evaluating the virial integral. The schematic of this approach is illustrated in Fig. 2. Shown is a cross-section of a cylindrical structure of Fig. 1, by the plane perpendicular to the axis of the cylinder. Imagine that we surround each star by a sphere of a radius $a_{\text {excl }}$, generally speaking different for different stars. The subscript "excl" stands for the word "exclusion." It is much greater than the radius of the star. The center of the sphere moves together with the star. Our whole approach works 
only if the global volume of the radius $R$ contains a large-enough number of stars, with the average star-to-star distance $l_{s t}$ satisfying condition $a_{\text {excl }}<<l_{s t}<<R$ (Fig. 2).

We denote the average (over the volume comprising a large number of stars) density of the stellar material as $\rho_{s t}$. The gravity acceleration produced by this mass density in some characteristic point of the global volume is of order of $g_{s t} \sim G R \rho_{s t}$.

This equation is correct if one does not come too close to a stellar object, see discussion below.

We require that, at the distance $a_{\text {excl }}$ from the star, the gravity acceleration $g_{\text {excl }}$, produced by the stellar object sitting in the center of the sphere of this radius be smaller than the global acceleration $g_{s t}$ defined by Eq. (10). Denoting the mass of an "average" star by $M_{s t}$, and the characteristic interstellar distance by $l_{s t}$, one can see that $M_{s t}$ is related to the average density of the stellar matter by $M_{s t} \sim \rho_{s t} l_{s t}^{3}$.

Accordingly, the acceleration $g_{\text {excl }} \sim G M_{s t} / a_{\text {excl }}^{2}$ at the surface of the exclusion sphere can be presented as

$$
g_{\text {excl }} \sim \frac{G \rho_{s t} l_{s t}^{3}}{a^{2}}
$$

and the condition $g_{\text {excl }}<g_{s t}$ acquires the form (Cf. Eq. (10)):

$\frac{a_{\text {excl }}}{l_{s t}}>\left(\frac{l_{s t}}{R}\right)^{1 / 2}$

Therefore, as one has $l_{s t}<<R$, one can always choose $a_{\text {excl }}$ to be much smaller than the inter-stellar distance $l_{s t}$. For the example that we consider in this paper, the spiral arm in our Galaxy, one has $l_{s t} \sim 2 \mathrm{pc}$, so that the right-hand side of Eq. (13), for $R=300 \mathrm{pc}$, is 0.1 . So, to satisfy the inequality (13) by some margin, one can take $a_{\text {exc }} \sim 0.3 l_{s t}$. With that, the exclusion volume is still quite small, $\sim 3 \%$ of the total volume.

Our basic approach is to include into the integration only the low-density interstellar medium, and describe gravitational effect on this medium from the stellar component by some average gravity acceleration, produced by the stellar matter "smeared" over the volumes containing many stars.

As we have already mentioned, individual stars have peculiar velocities $v_{\text {st }}$ of order of a few tens $\mathrm{km} / \mathrm{s}$. As the exclusion spheres have a relatively small radius, we have to move them together with the stars: otherwise, the star leaves the exclusion sphere within the time shorter than the "dynamic" time (1). Indeed, the time $a_{\text {excl }} / \mathrm{v}_{\mathrm{st}}$ for $a_{\text {excl }} \sim 0.6$ pc and $v_{\mathrm{st}} \sim 20 \mathrm{~km} / \mathrm{s}$ is mere $10^{4}$ years, orders of magnitude shorter than the dynamical time. Accordingly, we assume that every exclusion zone moves together with the star sitting in its center.

We perform now integration of Eq. (4) over the volume that excludes volumes of a radius $a_{\text {excl }}$ around the stars. We denote this volume by $\mathrm{V}_{\text {-, }}$ with the subscript "_.“ signifying that we do not include volumes around the stars. In this way we obtain, instead of Eq. (4):

$$
\int_{V_{-}} x_{\alpha} \frac{\partial \rho \mathrm{v}_{\alpha}}{\partial t} d V+\int_{V_{-}}\left(\rho \mathrm{v}^{2}+3 p+\frac{B^{2}}{4 \pi}\right) d V+\int_{V_{-}} \rho g_{\alpha} x_{\alpha} d V=Q
$$


$Q \equiv \sum_{i} \int_{S_{i}}\left(p \delta_{\alpha \beta}+\rho \mathrm{v}_{\alpha} \mathrm{v}_{\beta}+\frac{B^{2} \delta_{\alpha \beta}-B_{\alpha} B_{\beta}}{8 \pi}\right) x_{\alpha} d S_{\beta}$

The surface integrals are taken over the surfaces of the exclusion spheres, with the oriented surface elements $d \boldsymbol{S}$ directed away from the center. The subscript " $i$ " marks an individual stars inside the global volume of Fig. 1. The parameters in the L.H.S. are those of the interstellar medium. The gravity acceleration $\boldsymbol{g}$ in the L.H.S. includes that produced by the stellar matter.

When assessing the first term in the L.H.S. of Eq. (14), we have to remember that the integration domain $\mathrm{V}_{\text {- }}$ is ridden with exclusion zones which move in time. This means that one cannot just pull the partial time derivative out from the integrand as we did that when deriving Eqs. (7) and (8). One now has

$\int_{V_{-}} x_{\alpha} \frac{\partial \rho \mathrm{v}_{\alpha}}{\partial t} d V=\frac{d}{d t} \int_{V_{-}} \rho \mathrm{v}_{\alpha} x_{\alpha} d V-\int_{S_{i}} \rho \mathrm{v}_{\alpha} x_{\alpha}\left(\mathbf{u}_{i} \cdot d \mathbf{S}\right)$

where $\mathbf{v}_{i}$ is the peculiar velocity of the $i$ 'th star, generally speaking, varying with time. The surface integral should be taken over the instantaneous position of the surface, with an instantaneous value of the integrand.

Collecting several previous equations, we obtain that

$\frac{d}{d t} \int_{V_{-}} \rho \mathrm{v}_{\alpha} x_{\alpha} d V+\int_{V_{-}}\left(\rho \mathrm{v}^{2}+3 p+\frac{B^{2}}{4 \pi}\right) d V+\int_{V_{-}} \rho g_{\alpha} x_{\alpha} d V=Q+U$

where $Q$ is defined by Eq. (14), and

$U \equiv \sum_{i} \int_{S_{i}} \rho \mathrm{v}_{\alpha} x_{\alpha}\left(\mathbf{u}_{i} \cdot d \mathbf{S}\right)$

If the surface terms in the right-hand side (R.H.S.) of Eq.(17) were negligible, we would have obtained a desired equation, describing the equilibrium of the interstellar medium, with the effects from the large local contributions of the stellar matter eliminated.

Consider now one of the surface terms entering the quantity $Q$, Eq. (15), say, the term $\# i$. We present the radius-vector on the integration surface as

$\boldsymbol{r}=\boldsymbol{r}_{i}+\boldsymbol{r}_{i}^{\prime}$

where $\boldsymbol{r}_{i}$ is a radius-vector of the center of the compact object (drawn from the common, for all the stars, origin situated somewhere in the middle of the global structure), and $\boldsymbol{r}_{i}{ }^{\prime}$ is a vector connecting the center of the compact object with a particular point on the surface of a radius $a_{\text {excl }}$. Then, the surface integral $Q$ can be presented as:

$Q=Q^{(1)}+Q^{(2)}, Q^{(1,2)}=\sum_{i} Q_{i}^{(1,2)}$,

with

$Q_{i}^{(1)}=\int_{S_{i}}\left(p+\frac{B^{2}}{8 \pi}\right)\left(\boldsymbol{r}_{i} \cdot d \boldsymbol{S}\right)+\int_{S_{i}} \rho\left(\mathbf{v} \cdot \boldsymbol{r}_{i}\right)(\mathbf{v} \cdot d \boldsymbol{S})-\frac{1}{8 \pi} \int_{S_{i}} \rho\left(\boldsymbol{B} \cdot \boldsymbol{r}_{i}\right)(\boldsymbol{B} \cdot d \boldsymbol{S})$

$Q_{i}^{(2)}=a_{\text {excl }} \int_{S_{i}}\left(p+\frac{B_{t}^{2}}{8 \pi}\right) d S+a_{\text {excl }} \int_{S_{i}} \rho \mathrm{v}_{n}^{2} d S$

The subscripts " $n$ " and " $t$ " in Eq. (22) correspond to the components of the vectors normal and tangential to the surface of a sphere. 
When evaluating the integral $Q^{(l)}$, one has to remember that the vector $\boldsymbol{r}_{i}$ does not vary over the surface of the integration sphere. Then, the meaning of the integral becomes quite obvious: it is just the scalar product of $\boldsymbol{r}_{i}$ and the momentum $\dot{\boldsymbol{P}}_{i}$ imparted by the central object to the ISM per unit time, $Q_{i}^{(1)}=\boldsymbol{r}_{i} \cdot \dot{\boldsymbol{P}}_{i}$

Note, that the result does not depend on the exact value of the radius $a_{\text {excl }}$.

As there is no reason to believe that there is a correlation in the directions of $\dot{\boldsymbol{P}}_{i}$ for different stars, and the total number of stellar objects in a few $R$ long segment of the spiral arm is $\sim 3 \times 10^{7}$, the contributions from various stars cancel each other. So, we conclude that the sum of the terms $Q^{(I)}$ in Eq. (13) can be neglected. Statistical fluctuations whose relative value scales as $1 / \sqrt{N}$, where $N$ is the total number of stars in the volume under consideration, are small.

In a similar fashion, we conclude that the contributions of individual stars to the term $U$ (Eq. (18)) cancel each other, and this term can also be neglected.

Conversely, the terms $Q_{i}^{(2)}$ are sign-definite so that these contributions are added up and require a more careful evaluation. The exclusion zone is situated at a large distance from the star, a fraction of the inter-stellar distance (see Eq. (13)), where the gravitational contribution from the star becomes negligible. By noting that the surface of the exclusion volume is $4 \pi a_{\text {excl }}^{2}$, we conclude that, by the order of magnitude, the relative contribution of $\sum_{i} Q_{i}^{(2)}$ to Eq. (17) will be $a_{e x c l}^{3} / l_{s t}^{3} \sim\left(l_{s t} / R\right)^{3 / 2} \ll 1$ with respect to the L.H.S. of this equation. In other words, one can neglect this term. Thereby, the separation of the virial integral for the interstellar medium from the contribution from the stars is indeed feasible.

Of a concern is an effect of relatively rare high-energy events, like supernova explosions. For the segment of the spiral arm that we are considering, i.e., $\sim 300$ pc radius and a few radii long, supernova explosions occur, on average, once in $10^{4}$ years (Ferriere, 2001), releasing on average an energy of $10^{51} \mathrm{erg}$ (Arnett, 1996). This energy is comparable to the energy of the global magnetic field in the same volume. Supernovae explosions are often correlated to each other, i.e., several explosions occur in a relatively small volume within the time shorter than the average time (See Ferriere, 2001 for review and further references). This produces a strong jolt in the interstellar medium, leads to formation of "bubbles" and "superbubbles" surrounded by expanding shells of dense matter (see a nice succinct discussion of the phenomena involved in Bally, 1995). In the same directions act the clusters of bright young OB-type stars, which feature very intense outflows, losing tens of Solar masses within a few million years. All in all, these processes lead to a local variability with the time-scale less than or comparable to the dynamical time-scale (1). This may make the time-averaging procedure quite misleading. A question would inevitably arise as to whether the observed state is in any kind of equilibrium, or the state of the interstellar medium is highly dynamical, meaning that one cannot average out the time derivative in the L.H.S. of Eq. (17).

Despite these obvious problems with obtaining and interpreting the time-average virial integral, we proceed with its discussion - for reference purpose. In this way, by neglecting the R.H.S. in Eq. (17) and taking average over the time long compared to the dynamical time (1), we obtain: 


$$
\int_{V_{-}}\left(\rho \mathrm{v}^{2}+3 p+\frac{B^{2}}{4 \pi}\right) d V+\int_{V_{-}} \rho \boldsymbol{g} \cdot \boldsymbol{r} d V=0
$$

We skip here the averaging sign and remind that the integration is carried out over the entire volume, minus the exclusions zones around the stars.

\section{ATTEMPTING TO SEPARATE THE CONTRIBUTION OF DENSE CLOUDS}

When analyzing Eq. (23), one has to remember that, even after exclusion of the stars, the interstellar medium remains grossly non-uniform, with about $60 \%$ of the mass concentrated in dense clouds of molecular and atomic gas (Ferriere, 2001; Bregman, 2004; Heiles \& Crutcher, 2005). These clouds occupy approximately $10^{-2}$ of the volume of the spiral arm and have dimensions from $\sim 1$ parsec to tens of parsecs (Ferriere, 2001). The particle density in them is in the range of $50-10^{4} \mathrm{~cm}^{-3}$, and masses of individual clouds are between 100 and $10^{5}$ Solar masses. Individual clouds are often assumed to be in an internal virial equilibrium, where gravitational contraction is counterbalanced by the magnetic field pressure; it is possible that intense turbulence is present. The magnetic field plays a significant role in the dynamics of individual clouds and ranges between tens and many hundreds microgauss (this is to be compared with the average global magnetic field whose magnitude is thought to be a couple of microgauss, Beck et al, 2003; Han, 2006).

The virial integral (1) covers both the volume of high-density clouds and the much larger volume of the medium between them but is strongly dominated by the contribution of dense clouds. The clouds are relatively well-defined entities moving in the tenuous surrounding medium. Molecular clouds have three dimensional structure, whereas cold atomic clouds are probably more sheet-like (Kulkarni \& Heiles, 1988). A significant contribution to the integral in the form (24) comes from the kinetic energy associated with peculiar velocities of the clouds. So, the virial integral (24) is basically a sum of individual integrals over separate clouds and there is not much information regarding the behavior of these well-defined dense objects that can be obtained from the global virial integral (24). More useful will certainly be application of the virial theorem to individual clouds, as has been successfully done by McKee \& Zweibel, 1992. So, there seem to be not much utility in the integral written in the global form (24).

One may want, however, to isolate the virial integral for the remaining large volume filled by tenuous ionized gas (see Table 1 in Ferriere, 2001) permeated by a weak magnetic field of order of a couple of microgauss. It is tempting to repeat derivation of Sec. 3 by introducing new exclusion zones around the dense clouds, of a radius $a_{\text {excl }}$ several times larger than the scale of an individual cloud itself. The difficulties here arise from three circumstances: 1) The clouds are large and it is somewhat difficult to introduce non-overlapping exclusion zones: these zones occupy a significant part of the total volume; 2) The total number of clouds in the volume $\sim R^{3}$ is much smaller than the total number of stars (perhaps, $\sim 10^{3}-10^{4}$ vs $3 \times 10^{7}$ ), so that statistical errors becomes significantly larger, amplified by the fact that the mass of each cloud is large; 3) The clouds are "evaporating" under the action of ionizing radiation from the stars, especially from the nearby young stars; this makes the surface terms in Eq. (17) large.

We provide a more quantitative assessment of this last point, concentrating on $Q^{(2)}$, Eq. (22). By the order of magnitude, one can evaluate it as 
$a_{\text {excl }} \int_{S_{i}} \rho \mathrm{v}_{n}^{2} d S \sim a_{\text {excl }} \mathrm{V}\left|\dot{M}_{i}^{(\text {dense })}\right|$

where $M_{i}^{(\text {dense })}$ is the mass of an $i$ th isolated cloud. Eq. (25) characterizes the contribution of a single cloud; summing these contributions over all the clouds in the volume in question, one finds that

$Q^{(2)} \sim a_{\text {excl }} \mathrm{v} N_{\text {cloud }}\left|\dot{M}_{i}^{(\text {cloud })}\right|$,

where $N_{\text {cloud }}$ is the total number of dense clouds in the volume. For the purpose of qualitative estimates, we will describe the clouds as though all having some average radius $a_{\text {cloud }}$. The mass evaporation rate can be related to the average flux $F$ of the ionizing photons in the spiral arm. We are interested in the flux in terms of the number of ionizing photons passing through one square centimeter per second from a half-space $(2 \pi$ steradian). One has:

$\left|\dot{M}_{i}^{(\text {cloud })}\right| \sim 4 \pi a_{\text {cloud }}^{2} F m_{p}$,

where $m_{p}$ is the proton mass. Denoting by $\varepsilon$ a fraction of the total volume occupied by the clouds, one can state that

$\frac{4 \pi a_{\text {cloud }}^{3}}{3} N_{\text {cloud }} \sim \varepsilon \pi R^{2} L$.

Combining Eqs. (26)-(28), one obtains:

$Q^{(2)} \sim \frac{3 \varepsilon F a_{\text {excl }}}{a_{\text {cloud }}} \mathrm{vm} m_{p} \pi R^{2} L$

One can now compare this term with the kinetic energy contribution of the warm gas to the virial integral, which can be evaluated as, roughly, $I \sim \pi R^{2} L \times 5 T_{\text {warm }} n_{\text {warm }}$, accounting for both thermal and directional energy. The ratio of the two is:

$\frac{Q^{(2)}}{I} \sim \varepsilon \frac{3 a_{\text {excl }}}{5 a_{\text {cloud }}} \times \frac{\mathrm{vm}_{p} F}{n_{\text {warm }} T_{\text {warm }}}$.

For v we substitute $2 \times 10^{6} \mathrm{~cm} / \mathrm{s}$, that corresponds to the sound speed of $1 \mathrm{eV}$ plasma. We evaluate the average ionizing flux as $10^{8} \mathrm{~cm}^{-2} \mathrm{~s}^{-1}$, consistent with the average data presented in Ledoux and known examples of photo-evaporated molecular clouds, e.g., Hester et al, 1996; Pound, 1998; Levenson et al, 2000, Pound et al, 2003. For $n_{\text {warm }}$ we take $0.5 \mathrm{~cm}^{-3}$ and $T_{\text {warm }} \sim 1 \mathrm{eV}$. Finally, we assume that $3 a_{\text {excl }} / 5 a_{\text {cloud }} \sim 1$, and obtain that

$\frac{Q^{(2)}}{I} \sim 4$

This shows that the low-density warm matter is strongly coupled with massive clouds and its contribution to the virial integral cannot be isolated by the approach that was used for the stellar matter in Sec. 3 (under the assumption of insignificance of high-energy events).

Of course, in a quasi-steady state, the evaporation of dense clouds is balanced, on average, by formation of the new clouds via thermal and gravitational instabilities, sometimes triggered by high-energy events (Shapiro \& Field, 1976; for review see Bregman, 2004). But the contribution of this inverse process to $Q^{(2)}$ will also be positive, as is clearly seen from the general expression for $Q^{(2)}$. 


\section{DISCUSSION}

In conclusion: The validity of the virial integral for the interstellar medium is constrained by several requirements. First, there has to be a relatively well-defined and separated from the other parts of the Galactic disk "piece" of that disk, for example, a segment of the spiral arm. This requirement is related to that we need to consider an isolated system, as otherwise surface integrals appearing in the derivation turn out to be large and prevent from getting a clear-cut virial theorem.

The second constraint is related to the fact that the interstellar medium in this isolated piece of matter is coupled to the stellar population, which is by far dominant in the virial integral: the contributions of individual stars are localized on the scale of stellar radii and are orders of magnitude larger than the contribution of the interstellar matter. To isolate the latter, one can exclude the volume of the stars from the virial integral but then one is left with a sum of surface integrals taken over the surfaces of the "exclusion" volumes. For normal stars these integrals are relatively small, so that the separation of the interstellar matter from the stellar matter seems to be possible.

Difficulties, however, arise from the presence of rare energetic events, e.g., of supernova explosions, sometimes occurring in bursts. This gives rise to a significant uncertainty in the contribution to the surface integrals. Moreover, very large deviations from the average equilibrium may take place. This may affect the applicabilty of the virial theorem in its averaged form to the analysis of the observational data (which corresponds to a narrow time-interval).

If energetic events in a particular system are not important, then one can use a virial integral for interstellar medium, with contributions of the stars showing up only in the form of a gravitational potential produced by a smeared density distribution, Eq. (23). In this latter integral, however, the dominant role is played by dense neutral clouds, which are usually separated from each other and can be described as independent entities. Summing up their contributions does not add much insight into behavior of individual clouds which usually are in the virialized state. Dense clouds occupy a small frqction of the total volume, and one might want to isolate the contribution to the virial integral of the tenuous warm component. However, it turns out that the warm component is strongly coupled to the cold clouds and cannot be treated separately.

In other words, our final result is rather disappointing. However, the analysis carried out on the way to this conclusion can be of interest on its own, as it provides insights into inner workings of the virial theorem.

This work was performed under the auspices of the U.S. Department of Energy by University of California Lawrence Livermore National Laboratory under contract No. W7405-Eng-48. 


\section{REFERENCES}

Arnett, D. 1996 Supernovae and nucleosynthesis: an investigation of the history of matter, from the big bang to the present Princeton, NJ: Princeton University Press 1996

Bally J. 1995, In: The Physics and Chemistry of Interstellar Molecular Clouds, SpringerVerlag, Berlin-Heidelberg, p. 118.

Beck R, Shukurov A, Sokoloff D, Wielebinski R 2003 Astron. \& Astrophys. 41199.

Bregman JN. 2004 Astrophysics and Space Science, 289, 181.

Collins II, G.W. 2003 The Virial Theorem in Stellar Astrophysics. Electronic version: http://bifrost.cwru.edu/personal/collins/virial/

Crutcher, R.M. 1999, ApJ, 520, 706

Crutcher, R.M., Troland, T.H., Lazareff, B., Paubert, G., Kazes, I. 1999, ApJ, 514, L121

Crutcher, R.M. 2005. Observation of Magnetic Fields in Molecular Clouds - Testing Star Formation Paradigm, In: Magnetic Fields in the Universe: From Laboratory and Stars to Primordial Structures, American Institute of Physics, Conf. Proceedings \# 784.

Ferriere, K.M. 2001, Rev. Mod. Phys., 73, 1031

Han, J-L. 2006 J. of Phys., Conf. Ser., 47120

Heiles C, Crutcher R. Magnetic fields in diffuse H I and molecular clouds. Cosmic magnetic fields. Springer-Verlag. 2005, pp. 137-82. Berlin, Germany

Hester J.J., et al 1996. Astron.J., 111, 2349.

Lequeux, J. The Interstellar Medium (Springer, Berlin-Heidelberg-New York, 2005).

Levenson, N., Graham, J.R., McLean I.S., et al, ApJ, 533, L53.

McKee, C.F., Zweibel, E.G. 1992, ApJ, 399, 351

Pound, M.W. 1998 ApJ 493, L113

Pound, M.W., Reipurth, B., Bally, J. 2003, Astron. J., 125, 2108

Saslaw, W. C. 1985, Gravitational physics of stellar and galactic systems, Cambridge U. Press, Cambridge.

Shapiro, P.R., Field, G.B. 1976, ApJ, 205, 762. 
Spitzer, L. 1978, Physical Processes in the Interstellar Medium, N Y: Wiley-Interscience 1978 


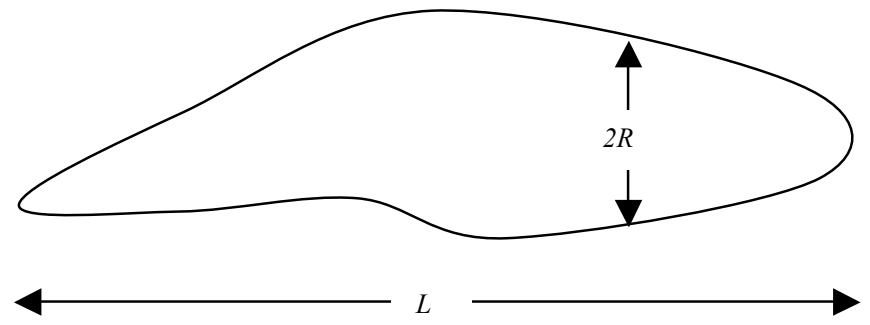

Fig. 1. A sketch of an "isolated" piece of the spiral arm, with $R \sim 300 \mathrm{pc}$, and $L \sim 1 \mathrm{kpc}$. 


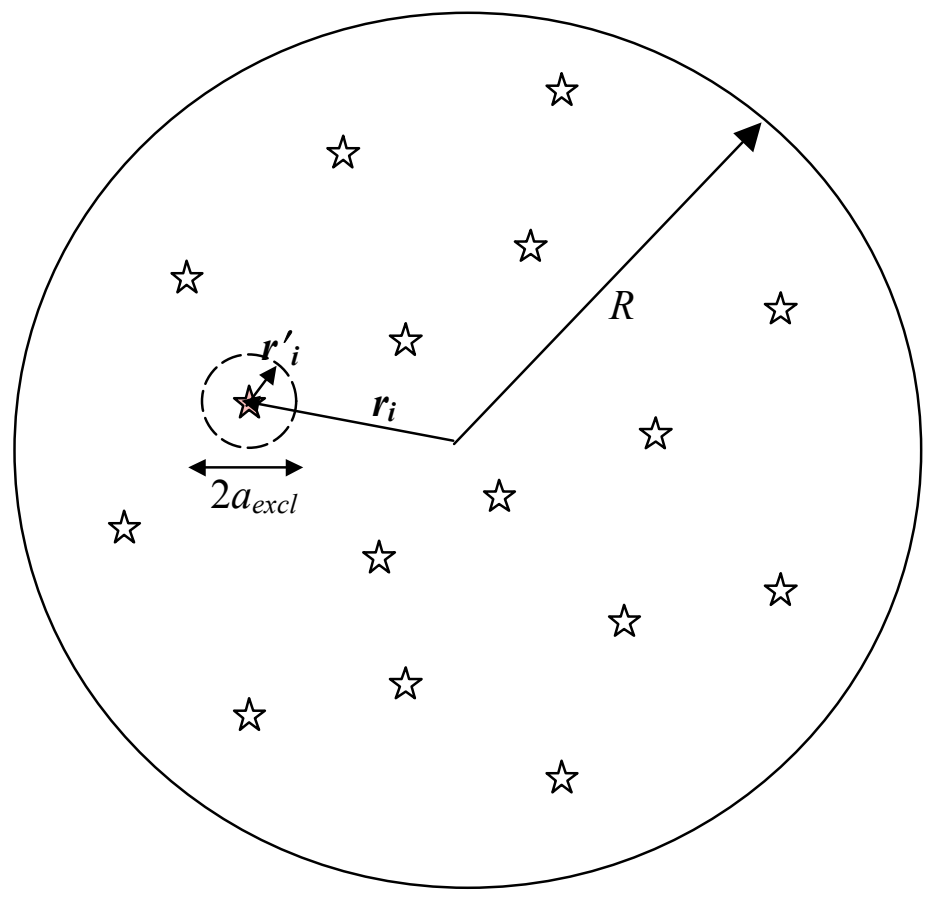

Fig. 2. On the definition of the exclusion sphere. Shown is a cross-section of a quasicylindrical volume of Fig. 1. The distance between neighboring stars is much greater than the radius of the exclusion zone (shown for one of the stars). 